\section{Estudo prospectivo da perda dentária em uma coorte de idosos dentados}

\author{
Prospective study on tooth loss in a cohort of \\ dentate elderly
}

\section{Estudio prospectivo de la pérdida dental en una cohorte de ancianos dentados}

\section{Resumo}

Este trabalho teve como objetivo avaliar fatores associados à perda dentária entre idosos de 60 anos e mais de idade num período de observação de quatro anos. Uma coorte de idosos dentados representativa da população da cidade de São Paulo, Brasil, participou do estudo. O desfecho foi a incidência de dentes perdidos entre os anos de 2006 a 2010. As variáveis independentes características demográficas, socioeconômicas, de uso e acesso a serviços de saúde, comportamento, morbidade referida, estado cognitivo, capacidade funcional, estado da dentição e uso de prótese foram medidas em 2006 e o desfecho em 2010. Utilizaram-se modelos de regressão binomial negativa. Participaram 440 indivíduos dentados. A análise final mostrou maior probabilidade de perda dentária em idosos que utilizavam duas próteses removíveis ( $R R=1,57$; IC95\%: 1,02-2,41), que avaliaram sua saúde bucal como regular ( $R R=1,62$; IC95\%: 1,11-2,36), ou ruim/ muito ruim ( $R R=1,87$; IC95\%: $1,11-3,17)$, do sexo masculino $(R R=1,74$; IC95\%: 1,28-2,37), e que moravam sozinhos ( $R R=2,03$; IC95\%: 1,11-3,72).

Perda de Dente; Serviços de Saúde Bucal; Idoso; Saúde Bucal
Doralice Severo da Cruz Teixeira 1,2

Paulo Frazão 1

Gizelton Pereira Alencar 1

Oswaldo Santos Baquero 3

Paulo Capel Narvai 1

Maria Lucia Lebrão 1

Yeda Aparecida de Oliveira Duarte 4
Correspondência D. S. C. Teixeira Faculdade de Saúde Pública, Universidade de São Paulo. Av. Dr. Arnaldo 715, São Paulo, SP 01246-904, Brasil. dsevero@uol.com.br uldade de Medicina Veterinária e Zootecnia, Universidade de São Paulo, ão Paulo, Brasil.

4 Escola de Enfermagem, Universidade de São Paulo, São Paulo, Brasil. 


\section{Introdução}

O envelhecimento populacional é uma conquista da humanidade, porém os anos conquistados devem ser vividos com qualidade. No que diz respeito à saúde bucal dos idosos, a perda dentária é um agravo de alta prevalência, impactando negativamente na manutenção da capacidade funcional.

A perda dentária constitui a expressão máxima de más condições de saúde bucal e representa o efeito cumulativo das doenças bucais. É resultante do nível de gravidade das doenças bucais, do modelo de atenção vigente e da maneira como as pessoas entendem o agravo ${ }^{1}$. Além de constituir uma marca de desigualdade social, causa problemas funcionais como a diminuição da capacidade mastigatória que limita o consumo de alimentos, afeta a fonação e a estética. Pode desencadear problemas psicológicos e sociais e, sobretudo, o edentulismo que é um fator de risco independente para a perda de peso que pode levar à fragilização do idoso, diminuindo ainda mais a sua qualidade de vida 2,3 .

Em 2010, os problemas bucais afetaram 3,9 bilhões de pessoas em todo o mundo, sendo que a cárie dentária, a periodontite e o edentulismo foram as condições mais prevalentes com valores de $35,4 \%, 10,8 \%$ e $2,3 \%$, respectivamente. Segundo o estudo sobre a carga global de doenças, o edentulismo ocupa o 81o lugar como causa de anos de vida perdidos ajustados por incapacidade (disability-adjusted life years - DALY), e entre os idosos, a perda dentária foi a principal causa de DALY 4,5. Um estudo realizado na Inglaterra encontrou o edentulismo independentemente associado com declínio físico e cognitivo em idosos e sugere que a perda dentária é um potencial marcador precoce de declínio em idades mais avançadas 6 .

Alguns países desenvolvidos apresentam uma tendência de declínio de perda dentária entre adultos, que se refletirá na população idosa nos próximos anos, mas as iniquidades sociais, no que diz respeito à saúde bucal, ainda persistem mesmo em países que têm programas avançados de saúde pública nesta área 7,8,9.

O estudo de Peres et al. 10, que compara os dados da Pesquisa Nacional de Saúde Bucal - SBBrasil realizada em 2002-2003 e em 2010 mostrou que as perdas dentárias entre idosos têm alta prevalência, sendo uma das maiores do mundo, e que $53,7 \%$ desta população são edêntulos (ausência total de dentes naturais). Embora entre adolescentes e adultos a magnitude da perda dentária esteja diminuindo, entre os idosos permanece estável. Assim, ao longo dos anos a perda continua ocorrendo, com a popu- lação idosa apresentando em média 25,3 dentes perdidos 11 .

A perda dentária é um importante problema de saúde pública no Brasil, em especial entre a população idosa, e se coloca como um desafio a ser enfrentado pelas políticas de saúde bucal de forma que as ações implantadas reduzam os danos causados por este agravo.

A hipótese deste estudo é que fatores associados ao envelhecimento também estejam associados à perda dentária, e sendo assim, serão possíveis as abordagens dos fatores comuns de risco para o enfrentamento do problema.

Desta forma, o objetivo deste trabalho foi identificar os fatores associados à perda dentária entre idosos de 60 anos e mais de idade em dois períodos de observação, correspondentes aos anos de 2006 e 2010.

\section{Métodos}

\section{Amostra e população do estudo}

Este é um estudo prospectivo que utilizou os dados provenientes de uma coorte de idosos dentados que iniciou no ano 2000 como resultado do Estudo SABE - Saúde Bem-Estar e Envelhecimento, de base populacional, multicêntrico, planejado e organizado pela Organização Pan-Americana da Saúde (OPAS), entre os anos de 1997 e 2000, em sete grandes cidades da América Latina e Caribe, cuja descrição detalhada do processo de obtenção da amostra encontra-se em outra publicação 12 .

Em 2000, participaram 2.143 pessoas de 60 anos e mais e, nesta primeira onda não foi realizado o exame clínico das condições bucais dos idosos. Assim, a linha de base do presente trabalho data de 2006, quando realizou-se a segunda onda e acrescentou-se uma nova coorte de idosos de 60-64 anos de idade. Foram sorteadas 394 pessoas, sendo que 298 participaram do estudo. Para o sorteio da nova coorte considerou-se uma taxa de prevalência para hipertensão arterial igual a $50 \%$, o efeito de delineamento igual a 1,5 e o erro de amostragem de $7 \%$. O tamanho da amostra foi corrigido para uma taxa de resposta igual a $75 \%$ e uma taxa de mortalidade de $2 \%$. A somatória das duas coortes, resultando em 1.413 idosos de 60 anos e mais, foi feita considerando-se ponderação de acordo com a taxa de mortalidade verificada para o intervalo entre as observações.

A terceira onda foi realizada entre 2010-2011 e incluiu 748 respondentes da coorte de 2000 e 242 da coorte de 2006. Entre 2006 e 2010 houve 288 óbitos (29,6\%), 10 institucionalizações (1\%), 
44 mudanças (4\%), 71 recusas $(7 \%)$ e $51(0,05 \%)$ idosos não foram localizados. Da mesma forma que em 2006, adicionou-se uma nova coorte de 355 pessoas entre 60 e 64 anos, totalizando 1.345 idosos com 60 anos e mais de idade.

Dessa forma, a amostra do presente estudo foi composta pelos participantes dentados examinados em 2006 e em 2010, que apresentaram informações para todas as variáveis explicativas utilizadas no modelo nos dois momentos de coleta, perfazendo um total de 446 pessoas (Figura 1).

O exame bucal foi realizado segundo o padrão constante no manual da Organização Mundial da Saúde (OMS) 13 por examinadores treinados e calibrados (a média do índice kappa para a concordância interexaminadores para cárie foi de 0,90 em 2006 e 0,87 em 2010). Adotou-se o valor de 0,65 como limite mínimo aceitável, à semelhança do utilizado no treinamento das equipes do levantamento epidemiológico SBBrasil 201011.

\section{Variáveis do estudo}

As variáveis utilizadas foram coletadas em $2006 \mathrm{e}$ o desfecho foi medido em 2010.

\section{- Variável dependente}

A variável dependente foi o número de dentes perdidos entre os anos de 2006 e 2010, calculada pela diferença entre o número de dentes presentes em 2006 e o número de dentes presentes em 2010. Os implantes, apoios das próteses fixas e as

\section{Figura 1}

Desenho do estudo. São Paulo, Brasil 2000-2006.

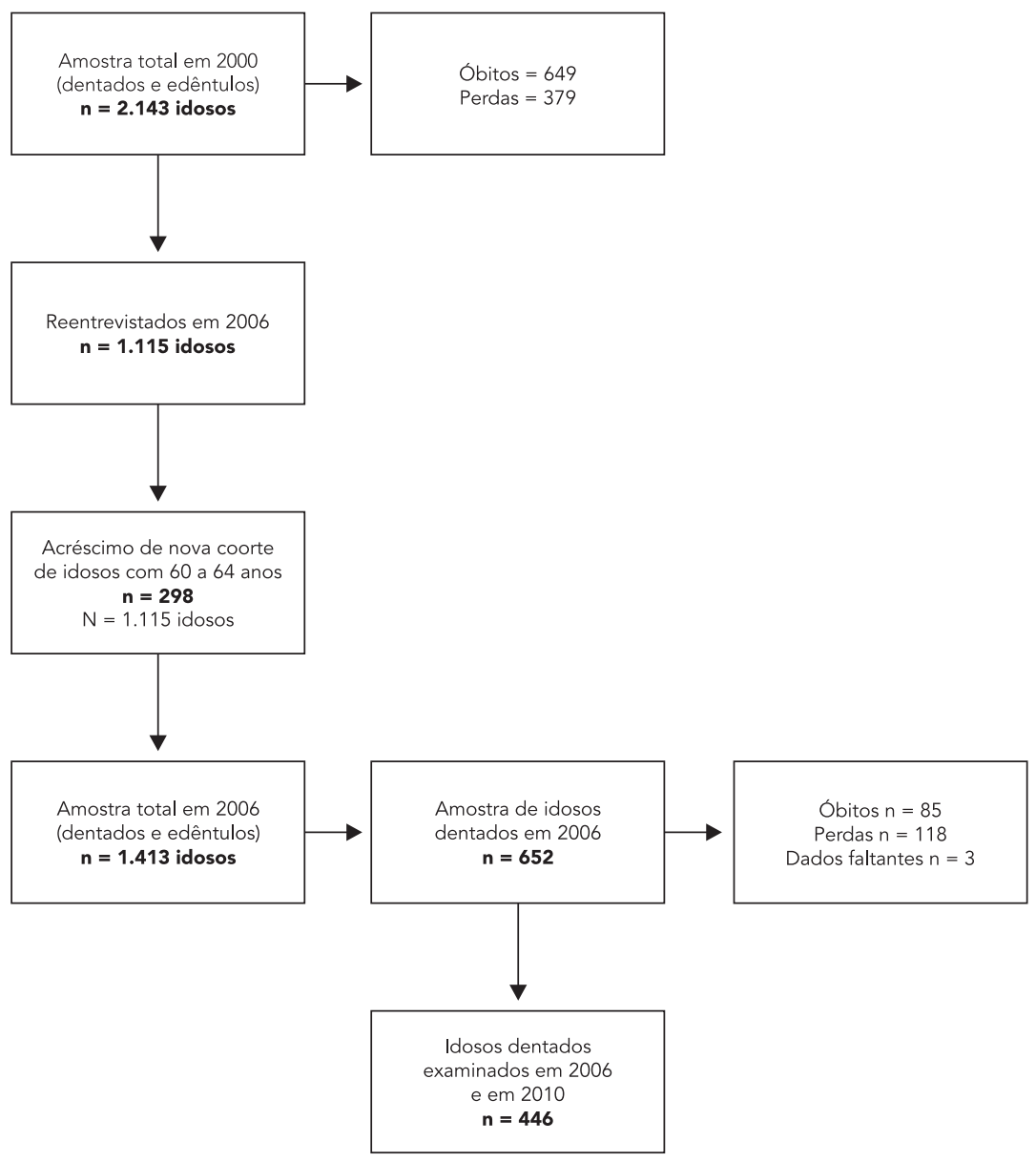


próteses fixas unitárias foram considerados como dentes presentes.

\section{- Variáveis independentes}

As variáveis independentes foram obtidas no ano de 2006 e compreenderam características sociodemográficas, de uso e acesso a serviços de saúde, comportamento, morbidade referida, estado cognitivo e capacidade funcional, estado da dentição e uso de prótese.

As variáveis sociodemográficas incluídas foram: sexo (homem, mulher); faixa etária distribuída em duas categorias, 60-74 anos de idade e 75 anos e mais no ano de 2006; situação de moradia (mora só: sim ou não); escolaridade (0-3 anos, 4-7, 8+ anos); autorrelato de insuficiência de renda para as despesas básicas (não, sim).

As variáveis de uso e acesso a serviços de saúde foram: utilização de outro plano de saúde além do sistema público, a data da última consulta ao dentista, se a mesma foi realizada nos últimos 12 meses anteriores à entrevista, entre 1 e 2 anos ou há mais de 3 anos, e o motivo dessa consulta: para tratamento, para manutenção ou para urgência.

Em relação ao comportamento, o hábito de fumar foi investigado e classificado em três categorias: nunca fumou; já fumou, mas não fuma mais; e fuma atualmente.

As variáveis relacionadas à morbidade autorreferida foram: presença ou não de diabetes mellitus, doença coronária, doença reumática, e ter ou não se sentido exausto na semana anterior à entrevista. Para avaliação do estado cognitivo dos idosos, utilizou-se uma versão modificada e validada no Chile do Mini Exame do Estado Mental (MEEM) 14, que teve o objetivo de reduzir o impacto da escolaridade sobre os resultados. Essa medida tem 13 itens que não dependem de escolaridade e uma pontuação total de 19 pontos. Assim, pontuação igual ou menor que 12 classificou o indivíduo como portador de déficit cognitivo.

A capacidade funcional foi avaliada por meio do comprometimento ou não das atividades básicas da vida diária (ABVD) que envolvem o autocuidado como alimentar-se, banhar-se, vestirse, arrumar-se, mobilizar-se, manter o controle dos esfíncteres e deambular; e das atividades instrumentais da vida diária (AIVD) que indicam a capacidade de um indivíduo levar uma vida independente dentro da comunidade como fazer compras, manipular medicamentos, administrar as próprias finanças e utilizar meios de transporte 15,16 .

O estado da dentição foi medido segundo o número de dentes ou coroas presentes em 2006 (valor mínimo 1 e valor máximo 28), o uso de prótese parcial removível (não usa, uso de prótese removível em um arco dentário, uso de prótese removível nos dois arcos dentários) e a autoavaliação de saúde bucal (boa/muito boa, regular, ruim/muito ruim). A variável número de dentes presentes em 2006 foi utilizada para ajustar as estimativas considerando-se as diferenças entre o número de dentes presentes na linha de base (2006) e o número de dentes presentes em 2010.

\section{Análise estatística}

Na construção do modelo, a associação entre a variável resposta e as variáveis explicativas foi testada inicialmente com uma regressão de Poisson. Dado que o valor do coeficiente de dispersão do modelo ajustado foi 2,53 $(>1,5)$ confirmando a superdispersão 17 , diferentes modelos lineares generalizados foram ajustados utilizando-se o teste de Vuong 18 e o Critério de Informação de Akaike-AIC 19; estes testes indicaram o modelo binomial negativo como a melhor opção.

Para evitar problemas de colinearidade utilizou-se o teste VIF (Variance Inflation Factor), e aquelas variáveis que apresentaram valores de inflação de variância inferiores a 5 foram mantidas no modelo final ${ }^{19}$, sendo que o modelo escolhido foi aquele que apresentou melhor valor de AIC.

As análises foram realizadas utilizando-se o ambiente de programação estatística $\mathrm{R}$ (The R Foundation for Statistical Computing, Viena, Áustria; http://www.r-project.org).

O efeito de cada variável independente sobre o desfecho foi estimado pela média de perda dentária e o respectivo intervalo de $95 \%$ de confiança (IC95\%), admitindo-se neste estudo como uma medida aproximada do risco relativo.

No modelo final, as variáveis explicativas foram número de dentes presentes, uso de prótese parcial removível, autoavaliação da saúde bucal, sexo, hábito de fumar, situação de moradia, sentimento de fadiga e idade.

\section{Considerações éticas}

O Estudo SABE foi aprovado pelo Comitê de Ética da Faculdade de Saúde Pública, Universidade de São Paulo, nos anos de 2006 e 2010, por meio dos Ofícios COEP 83/06 de 14 de março de 2006 e COEP 23/10 de 5 de maio de 2010, respectivamente. Os participantes assinaram o Termo de Consentimento Livre e Esclarecido no momento da entrevista, em que constavam informações detalhadas sobre a pesquisa e a forma de participação conforme Resolução no 196/1996 de 10 de outubro de 1996. 


\section{Resultados}

Participaram deste trabalho idosos dentados que foram examinados em 2006 e reexaminados em 2010, compreendendo um total de 446 indivíduos que possuíam dados para todas as variáveis explicativas utilizadas.

As características na linha de base (2006) dos entrevistados em 2010 foram comparadas com os que foram a óbito e com os que foram perdidos no período entre 2006 e 2010. Os que foram a óbito eram mais velhos, apresentavam maior dificuldade em ABVD e AIVD, pior desempenho cognitivo, relataram mais acidente vascular encefálico, doença pulmonar e não visitavam o dentista há mais de três anos quando comparados aos entrevistados $(\mathrm{p}<0,05)$. Os que foram perdidos no acompanhamento moravam sozinhos e possuíam menor número de dentes presentes do que os entrevistados $(\mathrm{p}<0,05)$ (dados não mostrados).

A média da idade da população estudada foi de 72,2 anos (IC95\%: 71,1-73,3). Dos participantes, $56 \%$ eram mulheres e $44 \%$ homens.

A Figura 2 apresenta os números de dentes perdidos e a proporção de idosos correspondente. Na população estudada, 54,9\% dos participantes não sofreram perda dentária e 45,1\% perderam um ou mais dentes, sendo que $19,6 \%$ perderam um dente e $25,5 \%$ perderam 2 ou mais. No período, $6,4 \%$ da população estudada tornaram-se edêntulas. A amplitude do número de dentes perdidos variou entre 1 e 17. A média de dentes perdidos no período foi de 1,34 ( $\mathrm{DP}=0,12$; IC95\%: 1,09-1,59). O desvio interquartílico foi de 0 a 11 dentes perdidos.

A média do índice CPOD (soma do número de dentes cariados, restaurados e perdidos) em 2006 foi de 23,5 (IC95\%: 22,9-24.1), sendo que o componente P registrou participação de 75,6\%. Em 2010, a média do Índice foi 25,0 (IC95\%: 24,325,6) e o componente $\mathrm{P}$ apresentou participação de $75,3 \%$.

A Tabela 1 mostra as características dos participantes. Entre os indivíduos dentados, a maioria estava na faixa etária de 60 a 74 anos, era do sexo feminino, com até 4 anos de estudos e declararou que sua renda era insuficiente para as necessidades básicas.

Mais de 50\% dos participantes foram ao dentista há mais de um ano. O motivo foi tratamento ou consulta de urgência (70\%).

Entre os entrevistados, 35,4\% informaram o hábito de fumar em idades mais jovens e 9,2\% se declararam fumantes no momento da visita.

O estado cognitivo estava comprometido em 8,1\% dos entrevistados, 9,6\% declararam ter dificuldade para a execução de uma ou mais ABVD e 37,9\% para a execução de uma ou mais AIVD.

As condições crônicas diabetes mellitus, doença reumática e doença coronária, todas elas autorreferidas, apresentaram uma prevalência de $16,3 \%, 31 \%$ e $21,2 \%$, respectivamente. O sentimento de exaustão foi declarado por $11,4 \%$ dos idosos entrevistados para este estudo.

Figura 2

Proporção de idosos segundo o número de dentes perdidos no período de 2006-2010. São Paulo, Brasil.

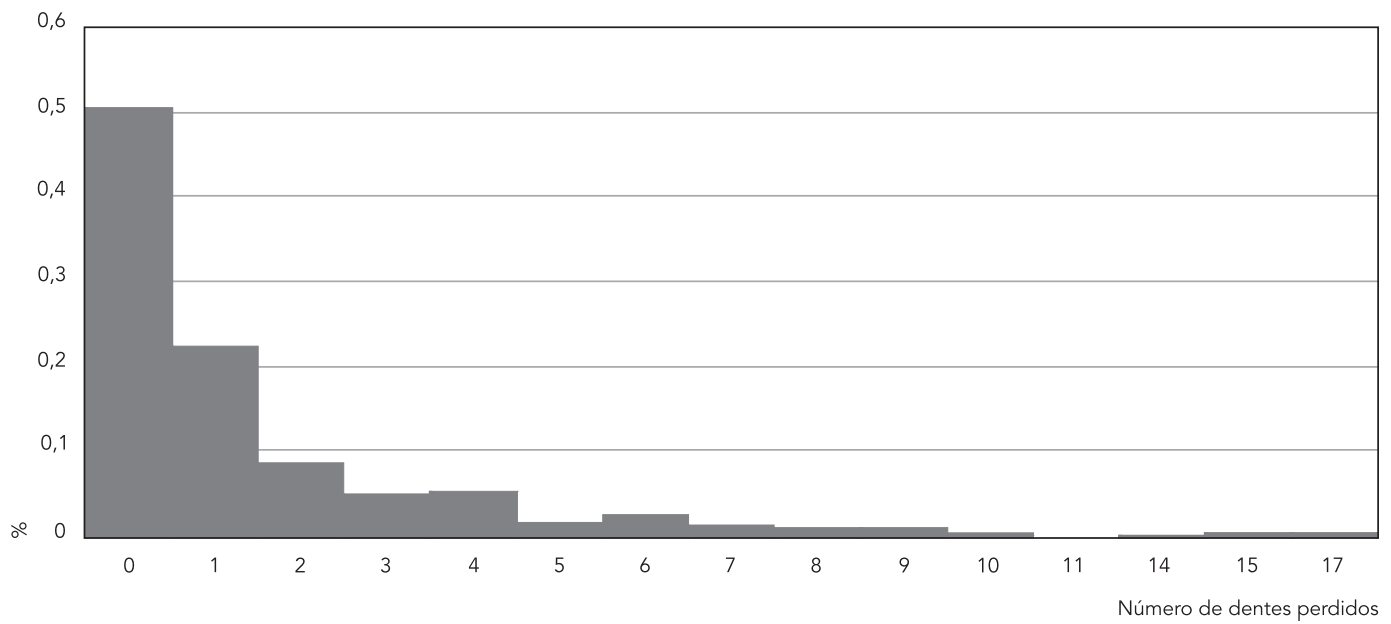


Tabela 1

Características da amostra de idosos dentados de 60 anos e mais de idade. Município de São Paulo, Brasil, 2006.

\begin{tabular}{|c|c|c|}
\hline Variável/Categorias & $\mathrm{n}$ & $\%$ \\
\hline \multicolumn{3}{|l|}{ Sexo } \\
\hline Mulher & 250 & 56,0 \\
\hline Homem & 196 & 44,0 \\
\hline \multicolumn{3}{|l|}{ Grupo etário (anos) } \\
\hline $75+$ & 135 & 13,3 \\
\hline $60-74$ & 311 & 86,7 \\
\hline \multicolumn{3}{|l|}{ Escolaridade (em anos) } \\
\hline $8+$ & 84 & 18,9 \\
\hline $5-8$ & 54 & 12,1 \\
\hline $0-4$ & 308 & 69,0 \\
\hline \multicolumn{3}{|c|}{ Situação de moradia (mora sozinho) } \\
\hline Não & 389 & 87,2 \\
\hline Sim & 57 & 12,8 \\
\hline \multicolumn{3}{|l|}{ Insuficiência de renda } \\
\hline Não & 203 & 45,4 \\
\hline Sim & 243 & 54,5 \\
\hline \multicolumn{3}{|c|}{ Data da última consulta ao dentista (anos) } \\
\hline Menos de 1 & 209 & 47,0 \\
\hline $1-2$ & 94 & 21,0 \\
\hline 3 ou mais & 143 & 32,0 \\
\hline \multicolumn{3}{|c|}{ Motivo da última consulta ao dentista } \\
\hline Manutenção & 126 & 28,2 \\
\hline Tratamento & 251 & 56,3 \\
\hline Urgência & 69 & 15,5 \\
\hline \multicolumn{3}{|c|}{ Utilização de outro plano de saúde além do SUS } \\
\hline Sim & 227 & 51,0 \\
\hline Não & 219 & 49,0 \\
\hline \multicolumn{3}{|l|}{ Hábito de fumar } \\
\hline Nunca fumou & 247 & 55,4 \\
\hline Já fumou, não fuma mais & 158 & 35,4 \\
\hline Fuma atualmente & 41 & 9,2 \\
\hline \multicolumn{3}{|l|}{ Diabetes melittus autorreferida } \\
\hline Não & 372 & 83,4 \\
\hline Sim & 74 & 16,6 \\
\hline \multicolumn{3}{|c|}{ Doença reumática autorreferida } \\
\hline Não & 299 & 67,0 \\
\hline Sim & 147 & 33,0 \\
\hline \multicolumn{3}{|l|}{ Doença coronária autorreferida } \\
\hline Não & 353 & 79,2 \\
\hline Sim & 93 & 20,8 \\
\hline \multicolumn{3}{|l|}{ Sentimento de exaustão } \\
\hline Não & 395 & 88,6 \\
\hline Sim & 51 & 11,4 \\
\hline \multicolumn{3}{|l|}{ Estado cognitivo * } \\
\hline 13 ou mais & 410 & 91,9 \\
\hline 12 ou menos & 36 & 8,1 \\
\hline
\end{tabular}

(continua) 


\begin{tabular}{|c|c|c|}
\hline Variável/Categorias & n & $\%$ \\
\hline \multicolumn{3}{|l|}{ Dificuldade para realizar ABVD } \\
\hline Não & 403 & 90,4 \\
\hline Sim & 43 & 9,6 \\
\hline \multicolumn{3}{|l|}{ Número de AIVD com dificuldade } \\
\hline 0 & 277 & 62,1 \\
\hline 1 & 62 & 13,9 \\
\hline 2 ou mais & 107 & 24,0 \\
\hline \multicolumn{3}{|l|}{ Autoavaliação da saúde bucal } \\
\hline Muito boa/Boa & 292 & 65,4 \\
\hline Regular & 110 & 24,7 \\
\hline Ruim/Muito ruim & 44 & 9,9 \\
\hline \multicolumn{3}{|l|}{ Uso de prótese } \\
\hline Não usa prótese & 251 & 56,3 \\
\hline Usa alguma prótese parcial removível & 141 & 31,6 \\
\hline Usa 2 próteses parciais removíveis & 54 & 12,1 \\
\hline \multicolumn{3}{|l|}{ Dentes presentes } \\
\hline Número mínimo & 1 & \\
\hline Número máximo & 28 & \\
\hline \multicolumn{3}{|l|}{ Perdeu algum dente entre 2006 e 2010} \\
\hline Não & 241 & 54,9 \\
\hline Sim & 221 & 45,5 \\
\hline
\end{tabular}

ABVD: atividades básicas da vida diária; AIVD: atividades instrumentais da vida diária.

Nota: Dados representativos de 399.553 idosos obtidos em 2006.

* Mini Exame do Estado Mental (MEEM), corte por escolaridade.

Dentre os participantes, $65,4 \%$ consideraram a sua saúde bucal boa ou muito boa, e $43,7 \%$ usavam alguma prótese parcial removível. O número máximo de dentes presentes foi 28.

A Tabela 2 mostra os valores brutos e ajustados do modelo final utilizando regressão binomial negativa. As variáveis de interesse não apresentaram fator de inflação de variância (VIF > 5) 19.

A análise final mostrou maior probabilidade de perda dentária em idosos que eram sexo masculino, que declararam morar sozinhos, que utilizavam duas próteses parciais removíveis, que avaliaram a sua saúde bucal como regular ou ruim/muito ruim. Sentir fadiga se mostrou como fator de proteção para o agravo. As variáveis faixa etária, hábito de fumar e número de dentes presentes na linha de base foram mantidas no modelo como variáveis de ajuste.

\section{Discussão}

Neste trabalho, os fatores associados à perda dentária em idosos foram sexo, morar sozinho, autoavaliar a saúde bucal como regular ou ruim/ muito ruim e utilizar duas próteses removíveis.
Comparadas com os homens, as mulheres apresentaram maior número de perdas dentárias 20,21,22,23. Neste estudo, os homens apresentaram maior probabilidade de perda. A razão entre $o$ número médio de perdas foi $1,74(\mathrm{p}=0,001)$. Esse achado pode ser atribuído ao fato de apresentarem mais dentes no início da pesquisa, pois as mulheres os perderam anteriormente, tendo em vista que os índices de cárie dentária são maiores desde a adolescência, o que pode explicar a diferença de perdas em idades mais avançadas 24 . Além disso, a perda dentária aumenta com a idade, o que pode explicar o risco maior entre os homens 25. O SBBrasil 2010 mostrou que as perdas dentárias diminuíram nas idades adultas, mas não entre os idosos 10,11.

Os idosos que declararam viver sozinhos apresentaram uma média de perda dentária 2,03 (IC95\%: 1,11-3,72; p = 0,022) maior do que aqueles que declararam viver acompanhados por outras pessoas. Um estudo longitudinal com uma coorte de idosos na cidade de Estocolmo, Suécia, mostrou que os que viviam sozinhos apresentaram maiores chances de cárie dentária quando comparados com os que viviam acompanhados $(\mathrm{OR}=2,4$; IC95\%: 1,2-7,2) 26. De fato, o apoio 
Tabela 2

Modelo final de regressão binomial negativa para fatores associados à perda dentária e média de perda dentária em uma coorte de idosos dentados no período de 2006-2010, segundo variáveis sociodemográficas, comportamento, morbidade autorreferida e saúde bucal (n = 446). Município de São Paulo, Brasil, 2010.

\begin{tabular}{|c|c|c|c|c|}
\hline \multirow{2}{*}{$\begin{array}{l}\text { Variáveis } \\
\text { Sexo (referência - mulher) }\end{array}$} & \multirow{2}{*}{$\begin{array}{l}\mathrm{RR} \text { * } \\
1,74\end{array}$} & \multicolumn{2}{|c|}{ IC95\% } & \multirow{2}{*}{$\begin{array}{c}\text { Valor de } p \\
0,001\end{array}$} \\
\hline & & 1,28 & 2,37 & \\
\hline Faixa etária (referência - 60-74 anos) & 1,04 & 0,76 & 1,44 & 0,769 \\
\hline Mora sozinho (referência - mora acompanhado) & 2,03 & 1,11 & 3,72 & 0,022 \\
\hline Ex-fumante (referência - nunca fumou) & 0,68 & 0,41 & 1,11 & 0,129 \\
\hline Fumante atual (referência - nunca fumou) & 1,79 & 0,89 & 3,22 & 0,050 \\
\hline Sentimento de exaustão (referência - não referiu exaustão) & 0,42 & 0,20 & 0,86 & 0,019 \\
\hline Uso de prótese parcial removível ** em 1 arco (referência - não usa prótese parcial removível) & 0,93 & 0,61 & 1,41 & 0,739 \\
\hline Uso de prótese parcial removível ** em 2 arcos (referência - não usa prótese parcial removível) & 1,57 & 1,02 & 2,41 & 0,037 \\
\hline Autopercepção da saúde bucal - regular (referência - autopercepção boa/muito boa) & 1,62 & 1,11 & 2,36 & 0,012 \\
\hline Autopercepção da saúde bucal - ruim/muito ruim (referência - autopercepção boa/muito boa) & 1,87 & 1,11 & 3,17 & 0,019 \\
\hline Número de dentes presentes & 1,01 & 0,98 & 1,05 & 0,223 \\
\hline
\end{tabular}

IC95\%: intervalo de 95\% de confiança; RR: risco relativo;

* RR estimado pela razão entre os valores médios de dentes perdidos;

** Ajustado pela faixa etária, hábito de fumar e número de dentes presentes.

social parece protegê-los por meio de ajuda e aconselhamento quando necessário 27,28 . Idosos que moram sozinhos deveriam ser apoiados pelo Estado e pela comunidade. Os serviços de saúde deveriam se preparar para dar o suporte necessário a essas pessoas. Novos arranjos e serviços inovadores deveriam ser criados para dar conta do número crescente de pessoas nessa situação.

Os idosos que declararam sentimento de fadiga na semana anterior à visita apresentaram $58 \%$ menos dentes perdidos quando comparados aos demais 0,42 (IC95\%: 0,20-0,86; p = 0,019), indicando que o sentimento de esgotamento pode ser um fator de proteção para o agravo e que de alguma forma estavam recebendo cuidado. $\mathrm{Na}$ amostra estudada, $10 \%$ dos idosos declararam sensação de fadiga, sendo que dentre estes, $23 \%$ tinham cuidador. A ajuda recebida pelos idosos por intermédio dos cuidadores pode se estender à higiene bucal, o que seria uma explicação para o resultado. Não sendo então o sentimento de fadiga a proteger os idosos da perda dentária, mas sim a presença do cuidador auxiliando-os na higiene pessoal.

A sensação de esgotamento é um dos sintomas da síndrome da fragilidade que é um problema vitalmente importante no tratamento dos idosos. A fragilidade é uma síndrome que resulta do envelhecimento e da multimorbidade, e pode afetar fortemente como uma pessoa idosa vai responder ao tratamento médico, bem como quanto tempo e quão bem viverá 29 . As equipes de saúde bucal devem estar preparadas para reconhecer os sinais da síndrome no dia a dia da clínica, a fim de ofertar o tratamento oportuno buscando a manutenção da capacidade funcional.

A autoavaliação de saúde bucal foi um forte preditor de perda dentária. O valor médio de dentes perdidos foi 1,62 (IC95\%: 1,11-2,36; $\mathrm{p}=$ 0,012 ) vez maior para a autoavaliação regular e 1,87 (IC95\%: 1,11-3,17; p = 0,019) vez maior para a autoavaliação ruim/muito ruim. Os resultados apresentam um gradiente importante mostrando que a autoavaliação da saúde bucal é um dado que deve ser conhecido pelas equipes que promovem o cuidado a essa população. A autoavaliação ruim das condições de saúde bucal parece ser preditora de desfechos indesejados. Tal como na saúde geral, pessoas que relatam a sua saúde bucal como ruim podem apresentar maior risco de perda dentária, dado que a autopercepção está associada fortemente com o estado real ou objetivo de saúde das pessoas e pode ser encarada como uma representação das avaliações objetivas de saúde, e a perda dentária ser o desfecho indesejado da maioria das doenças e condições dentárias 30,31.

Assim, além das medidas normativas, as equipes de saúde bucal devem levar em conta as medidas subjetivas que poderão auxiliar no momento do planejamento das ações no território.

Os idosos que utilizavam duas próteses parciais removíveis apresentaram uma perda dentária 1,57 (IC95\%: 1,02-2,41; $\mathrm{p}=0,037$ ) vez maior 
quando comparados aos que não utilizavam. Estudos longitudinais têm mostrado que os dentes utilizados como apoio de próteses parciais removíveis estão significativamente relacionados com a progressão da doença periodontal e a consequente perda dentária $32,33,34$

Um estudo realizado na Europa mostrou que o uso de todos os tipos de próteses dentárias foi altamente prevalente, especialmente entre os grupos etários mais velhos 35 . Dados do levantamento epidemiológico SBBrasil 2003 indicaram que $66,54 \%$ dos idosos brasileiros usavam algum tipo de prótese no arco superior e 42,57\% no inferior. No tocante à necessidade de prótese, $30,96 \%$ das pessoas examinadas precisavam de algum tipo de prótese superior e 54,52\% de inferior 36. O levantamento epidemiológico SBBrasil 2010 mostrou que 76,5\% dos idosos usavam algum tipo de prótese no arco superior e $53,9 \%$ no inferior 11 . Os dados indicam que o acesso a esse dispositivo aumentou nos últimos 10 anos, especialmente pelas políticas públicas oferecidas desde 2004, por meio do Programa Brasil Sorridente.

A avaliação da necessidade de prótese foi realizada por cirurgiões-dentistas segundo os critérios de retenção, estabilidade, fixação e estética. Quando pelo menos uma dessas condições estava presente, recomendava-se a sua substituição. Observou-se que 92,7\% dos idosos necessitavam um ou ambos os arcos dentários e, dentre estes, $54,3 \%$ tinham a necessidade de próteses parciais removíveis. Os dados mostram a magnitude das perdas dentárias nessa população.

De acordo com os levantamentos epidemiológicos nacionais, a necessidade de próteses dentárias no Brasil é alta e estes dispositivos deveriam ser confeccionados de forma a proporcionar a melhora da saúde bucal sem risco de iatrogenias. O sistema público de saúde brasileiro (Sistema Único de Saúde - SUS) oferta próteses totais e parciais removíveis. Os municípios deveriam se organizar para ofertar o procedimento para a população e as equipes deveriam estar preparadas para tal. A prótese dentária pode ser um importante dispositivo de recuperação dos danos que acometem a saúde bucal das pessoas ao longo da vida, se os portadores forem assistidos periodicamente.

Pesquisas têm mostrado que os fatores sociais são elementos centrais na determinação do padrão de morbimortalidade, na adoção de hábitos saudáveis e na distribuição dos serviços e recursos de saúde 37,38 .

Destaca-se que o resultado encontrado por este estudo no que diz respeito ao risco de perda dentária por uso de duas próteses removíveis, acrescenta mais um item no cuidado em saúde bucal dos idosos. As perdas dentárias ocorrem ao longo da vida e são determinadas pelo nível socioeconômico, baixos níveis educacionais e de renda, e representando, sobretudo na população idosa, o efeito cumulativo das doenças bucais.

Peres et al. 10 compararam os dados das Pesquisas Nacionais de Saúde Bucal realizadas em 2002-2003 e 2010, e encontraram maior prevalência de perda dentária entre os idosos de menor renda e escolaridade. No presente estudo não foram registradas associações com essas variáveis. Uma possível explicação pode ser o fato de que os idosos que apresentam maior retenção dentária são aqueles com maior renda e escolaridade, e a amostra utilizada foi de idosos dentados.

Metade da população de idosos dentados $(49,3 \%)$ teve algum dente perdido no período de quatro anos. Num estudo sobre a incidência da perda dentária entre idosos na cidade de Carlos Barbosa, Rio Grande do Sul, os pesquisadores encontraram um valor de $67,8 \%$ num período de seguimento de 4 anos 39 . Assim, a perda dentária varia dentro do país e apresenta valores mais altos do que os encontrados em outros lugares do mundo ${ }^{10}$. Uma revisão sistemática da literatura realizada em 2007 sobre a incidência de perda dentária em países da Europa encontrou valores entre $1 \%$ e $14 \%{ }^{40}$. No Japão, na cidade de Niigata, a perda dentária entre idosos, num período de seguimento de cinco anos, foi de $6,7 \% 41$.

O envelhecimento da população traz consigo a necessidade da organização dos serviços para enfrentar a carga de doenças que o acompanha, e neste contexto está inserida a saúde bucal que por ser parte integrante da saúde geral não deveria ser negligenciada, sob pena de prejudicar os esforços para assegurar melhor qualidade de vida para a população idosa.

A hipótese de que fatores associados ao envelhecimento também estivessem associados à perda dentária não foi confirmada neste trabalho, porém os resultados encontrados podem contribuir para a produção de conhecimentos que subsidiem a formulação de programas voltados ao controle da perda dentária entre idosos, considerando que a garantia de acesso e o uso dos serviços para esta população continua a ser um desafio no planejamento de cuidados de saúde bucal, e que a perda dentária afeta negativamente o bem-estar e a qualidade de vida $42,43,44,45$.

Há ainda alguns pontos importantes a serem considerados em relação a esta pesquisa. Por tratar-se de um estudo longitudinal, a representatividade é referida à linha de base fixada em 2006. Nessa data, a amostra era representativa dos idosos do município sempre que devidamente ponderada pelos pesos decorrentes do desenho 
amostral. Os idosos avaliados em 2010 são representativos dos sobreviventes daquela população. O segundo ponto a considerar é que os fatores foram medidos em 2006 e o desfecho em 2010, permitindo a mensuração do risco a que estão submetidos os idosos.

Uma das fragilidades deste estudo é o uso de dados autorrelatados sobre condições crônicas. Embora possa ser uma fonte de viés, outros pesquisadores têm mostrado que a autoavaliação de saúde é consistente com diagnósticos médicos 46 . Outra fragilidade é a ausência de variáveis de hábitos sobre higiene bucal e dieta cariogênica.

$\mathrm{O}$ estudo mostrou que os fatores associados à perda dentária foram ser do sexo masculino, morar sozinho, usar prótese parcial removível nos dois arcos e autoavaliar a saúde bucal como regular ou ruim/muito ruim. O hábito de fumar perdeu significância na análise ajustada, porém é um dos fatores comuns de risco para várias doenças, e as abordagens para a cessação deste hábito devem ser realizadas pelas equipes de saúde bucal. A compreensão da influência de cada fator na perda dentária permitirá o estabelecimento de estratégias voltadas para a população idosa e para a maneira como está ocorrendo o envelhecimento, com o objetivo de diminuir este agravo e favorecer a qualidade de vida desta população.

Esses esforços serão mais consistentes quanto maior for a base de conhecimentos científicos sobre o processo saúde-doença e a adoção de abordagens transdisciplinares no atendimento dessa população.

\section{Colaboradores}

D. S. C. Teixeira participou da concepção do artigo, de todas as etapas da produção e foi responsável pela versão final. P. Frazão contribuiu na concepção do projeto e revisão crítica do conteúdo. G. P. Alencar e O. S. Baquero participaram das etapas de produção do artigo e análises estatísticas. P. C. Narvai, M. L. Lebrão e Y. A. O. Duarte contribuíram na revisão crítica do conteúdo do artigo.

\section{Referências}

1. Roncalli AG, Barbato PR, Resende CMBM. Perdas dentárias. In: Antunes JLF, Peres MA. organizadores. Epidemiologia da saúde bucal. 2ạ Ed. São Paulo: Editora Santos; 2013. p. 335-53.

2. Marcenes W, Steele JG, Sheiham A. The relationship between dental status, food selection, nutrient intake, nutritional status, and body mass index in older people. Cad Saúde Pública 2003; 19: 809-16.

3. Lee JS, Weyant RJ, Corby P, Kritchevsky SB, Harris TB, Rooks R, et al. Edentulism and nutritional status in a biracial sample of well functioning, community-dwelling elderly: the Health, Aging, and Body Composition Study. Am J Clin Nutr 2004; 79:295-302.

4. Vos T, Flaxman AD, Naghavi M, Lozano R, Michaud C, Ezzat M. Years lived with disability (YLDs) for 1160 sequelae of 289 diseases and injuries 19902010: a systematic analysis for the Global Burden of Disease Study 2010. Lancet 2012; 380:2163-96.

5. Marcenes W, Kassebaum NJ, Bernabé E, Flaxman A, Naghavi M, Lopez A, et al. Global burden of oral conditions in 1990-2010: a systematic analysis. J Dent Res 2013; 92:592-7.

6. Tsakos G, Watt RG, Rouxel PL, Oliveira C, Demakakos P. Tooth loss associated with physical and cognitive decline in older adults. J Am Geriatr Soc 2015; 63:91-9.

7. Petersen PE, Kjarller M, Christensen LB, Krustrup U. Changing dentate status of adults, use of dental health services, and achievement of National Dental Health Goals in Denmark by the year 2000. J Public Health Dent 2004; 64:127-35. 
8. Petersen PE, Yamamoto T. Improving the oral health of older people: the approach of the WHO Global Oral Health Programme. Community Dent Oral Epidemiol 2005; 33:81-92.

9. Bernabé E, Marcenes W. Income inequality and tooth loss in the United States. J Dent Res 2011; 90:724-9.

10. Peres MA, Barbato PR, Reis SC, Freitas $\mathrm{CH}$, Antunes JL. Tooth loss in Brazil: analysis of the 2010 Brazilian Oral Health Survey. Rev Saúde Pública 2013; 47 Suppl 3:78-89.

11. Secretaria de Atenção à Saúde; Secretaria de Vigilância em Saúde, Ministério da Saúde. SB Brasil 2010: Pesquisa Nacional de Saúde Bucal: resultados principais. Brasília: Ministério da Saúde; 2012.

12. Lebrão ML, Laurenti R. Saúde, bem-estar e envelhecimento: o estudo SABE no Município de São Paulo. Rev Bras Epidemiol 2005; 8:127-41.

13. World Health Organization. Oral health surveys: basic methods. $4^{\text {th }}$ Ed. Geneva: World Health Organization; 1997.

14. Icaza MC, Albala C. Projeto SABE. Minimental State Examination (MMSE) del estudio de dementia en Chile: análisis estatístico. Washington DC: Pan-American Health Organization; 1999.

15. Katz S, Ford AB, Moskowitz RW, Jackson BA, Jaffe MW. Studies of illness in the aged. The index of ADL: a standardized measure of biologycal and psychosocial function. JAMA 1963; 185:914-9.

16. Lawton MP, Brody EM. Assessment of older people: self-maintaining and instrumental activities of daily living. Gerontologist 1969; 9:179-86.

17. Matthiopoulos J. How to be a quantitative ecologist: the 'A to R' of green mathematics and statistics. Chichester: John Wiley \& Sons; 2011.

18. Vuong QH. Likelihood ratio tests for model selection and non-nested hypotheses. Econometrica 1989; 57:307-33.

19. Zuur A, Leno EN, Walker N, Savelier AA, Smith GM Mixed effects models and extensions in ecology with R. New York: Springer; 2009.

20. Yoshino K, Ishizuka Y, Watanabe H, Fukai K, Sugihara N, Matsukubo T. Sex-and age-based differences in single tooth loss in adults. Bull Tokyo Dent Coll 2015; 56:63-7.

21. Mesas AE, Andrade SM, Cabrera MAS. Oral health status of community-dwelling elderly in Londrina, Paraná, Brazil. Rev Bras Epidemiol 2006; 9:471-80.

22. Corraini P, Baelum V, Pannuti CM, Pustiglioni NA, Romito GA, Pustiglioni FE. Tooth loss prevalence and risk indicators in an isolated population of Brazil. Acta Odontol Scand 2009; 67:297-303.

23. Lukacs JR. Gender differences in oral health in South Asia: metadata imply multifactorial biological and cultural causes. Am J Hum Biol 2011; 23:398-411.

24. Peres MA, Peres KG, Barros AJD, Victora CG. The relationship between family socioeconomic trajectories from childhood to adolescence and dental caries and associated oral behaviours. J Epidemiol Community Health 2007; 61:141-5.

25. Saliba NA, Moimaz SAS, Saliba O, Tiano AVP. Perda dentária em uma população rural e as metas estabelecidas pela Organização Mundial de Saúde. Ciênc Saúde Coletiva 2010; 15 Suppl 1:1857-64.
26. Avlund K, Holm-Pedersen P, Morse DE, Viitanen M, Winblad B. Social relations as determinants of oral health among persons over the age of 80 years. Community Dent Oral Epidemiol 2003; 31:454-62.

27. Locker D, Ford J, Leake JL. Incidence of and risk factors for tooth loss in a population of older Canadians. J Dent Res 1996; 75:783-9.

28. Hanson BS, Liedberg B, Öwall B. Social network, social BS, support and dental status in elderly Swedish men. Community Dent Oral Epidemiol 1994; 22(5 Pt 1):331-7.

29. Fried LP, Tangen CM, Walston J, Newman AB, Hirsch C, Gottdiener J, et al. Frailty in older adults: evidence for a phenotype. J Gerontol A Biol Sci Med Sci 2001; 56:M146-56.

30. Dolan TA, Gilbert GH, Duncan RP, Foerster U. Risk indicators of edentulism, partial tooth loss and prosthetic status among black and white middleaged and older adults. Community Dent Oral Epidemiol 2001; 29:329-40.

31. Raja BK, Radha G. Self-perceived oral function and factors influencing oral health of elderly residents in Bengaluru city, India. Journal of Health Research and Reviews 2015; 2:29-33.

32. Hiritomi T, Yoshihara A, Yano M, Ando Y, Miyazaki H. Longitudinal study on periodontal conditions in healthy elderly people in Japan. Community Dent Oral Epidemiol 2002; 30:409-17.

33. Preshaw PM, Walls AW, Jakubovics NS, Moynihan PJ, Jepson NJ, Loewy Z. Association of removable partial denture use with oral and systemic health. J Dent 2011; 39:711-9.

34. Hussain KA, Azzeghaibi SN, Tarakji B, SenthilRajan RS, Sirajuddin S, Prabhu SS. Iatrogenic damage to the periodontium caused by removable prosthodontic treatment procedures: an overview. Open Dent J 2015; 9:187-9.

35. Zitzmann NU, Hagmann E, Weiger R. What is the prevalence of various types of prosthetic dental restorations in Europe? Clin Oral Implants Res 2007; 18 Suppl 3:20-33.

36. Departamento de Atenção Básica, Secretaria de Atenção à Saúde, Ministério da Saúde. Projeto SB Brasil 2003: condições de saúde bucal da população brasileira 2002-2003: resultados principais. Brasília: Ministério da Saúde; 2004.

37. Barros MBA, Francisco PMSB, Zanchetta LM, Cesar CLG. Tendências das desigualdades sociais e demográficas na prevalência de doenças crônicas no Brasil, PNAD: 2003-2008. Ciênc Saúde Coletiva $2011 ; 16: 3755-68$.

38. Travassos C, Castro MSM. Determinantes e desigualdades sociais no acesso e na utilização de serviços de saúde. In: Giovanella L, Escorel S, Lobato LVC, Noronha JC, Carvalho AI, organizadores. Políticas e sistema de saúde no Brasil. Rio de Janeiro: Editora Fiocruz; 2008. p. 215-43.

39. De Marchi RJ, Hilgert JB, Hugo FN, dos Santos CM, Martins AB, Padilha DM. Four-year incidence and predictors of tooth loss among older adults in a southern Brazilian city. Community Dent Oral Epidemiol 2012; 40:396-405.

40. Müller F, Naharro M, Carlsson GE. What are the prevalence and incidence of tooth loss in the adult and elderly population in Europe? Clin Oral Implants Res 2007; 18 Suppl 3:2-14. 
41. Hirotomi T, Yoshihara A, Ogawa H, Miyazaki H. Tooth-related risk factors for tooth loss in community-dwelling elderly people. Community Dent Oral Epidemiol 2012; 40:154-63.

42. Smith JM, Sheiham A. How dental conditions handicap the elderly. Community Dent Oral Epidemiol 1979; 7:305-10.

43. Davis DM, Fiske J, Scott B, Radford DR. The emotional effect of tooth loss: a preliminary quantitative study. Br Dent J 2000;188:503-6.

44. Ferreira CO, Antunes JLF, Andrade FB. Factors associated with the use of dental services by elderly Brazilians. Rev Saúde Pública 2013; 47 Suppl 3:1-7.
45. Hugo FN, Hilgert JB, da Luz Rosário de Souza M, Cury JA. Oral status and its association with general quality of life in older independent-living southBrazilians. Community Dent Oral Epidemiol 2007; 37:231-40.

46. Reuben DB, Siu AL, Kimpau S. The predictive validity of self-reported and performance-based measures of function and health. J Gerontol 1992; 47:M106-10.

\begin{abstract}
The aim of this study was to assess factors associated with tooth loss in elderly 60 years or older during a four-year observation period. A representative cohort of dentate elderly from the city of São Paulo, Brazil, participated in the study. The outcome was teeth loss incidence from 2006 to 2010. Demographic and socioeconomic characteristics, health services access and use, behavior, reported diseases, cognitive status, functional status, state of dentition, and use of dental prosthesis were recorded as independent variables in 2006 and the outcome was measured in 2010. Negative binomial regression models were used. Participation included 440 dentate elderly. Increased likelihood of tooth loss was associated with use of two removable prostheses $(R R=1.57 ; 95 \% C I: 1.02-2.41)$, fair self-rated oral health ( $R R=1.62 ; 95 \% C I: 1.11-2.36)$, bad/very bad self-rated oral health ( $R R=1.87 ; 95 \% C I$ : 1.11-3.17), male gender ( $R R=1.74 ; 95 \% C I: 1.28-2.37)$, and living alone $(R R=2.03$; 95\%CI: 1.11-3.72).
\end{abstract}

Tooth Loss; Dental Health Services; Aged; Oral Health

\section{Resumen}

Este estudio tuvo como objetivo evaluar factores asociados a la pérdida dental entre ancianos de 60 años $y$ más de edad, en un período de observación de 4 años. Participó en el estudio una cohorte de ancianos dentados, representativa de la población de la ciudad de São Paulo, Brasil. El resultado fue la incidencia de dientes perdidos entre los años de 2006 y 2010. Se midieron en 2006 las variables independientes, características demográficas, socioeconómicas, de uso y acceso a servicios de salud, comportamiento, morbilidad referida, estado cognitivo, capacidad funcional, estado de la dentición y el uso de prótesis. Se analizó el resultado en 2010. Se utilizaron modelos de regresión binomial negativa. Participaron 440 individuos dentados, cuyo análisis final mostró mayor probabilidad de pérdida dental en ancianos que utilizaban dos prótesis removibles $(R R=1,57$; IC95\%: 1,02-2,41), que evaluaron su salud bucal como regular ( $R R=1,62$; IC95\%: 1,112,36) o mala/muy mala ( $R R=1,87$; IC95\%: 1,11-3,17), del sexo masculino $(R R=1,74$; IC95\%: 1,28-2,37) y que vivían solos (RR = 2,03; IC95\%: 1,11-3,72).

Pérdida de Diente; Servicios de Salud Dental; Anciano; Salud Bucal
Recebido em 06/Fev/2015

Versão final reapresentada em 03/Jun/2015 Aprovado em 18/Fev/2016 